\title{
Influence of electron recapture by the cathode upon the discharge characteristics in dc planar magnetrons
}

\author{
Ivan Kolev, ${ }^{*}$ Annemie Bogaerts, and Renaat Gijbels \\ Department of Chemistry, University of Antwerp, Universiteitsplein 1, 2610 Wilrijk, Belgium \\ (Received 31 July 2004; revised manuscript received 13 June 2005; published 18 November 2005)
}

\begin{abstract}
In dc magnetrons the electrons emitted from the cathode may return there due to the applied magnetic field. When that happens, they can be recaptured or reflected back into the discharge, depending on the value of the reflection coefficient ( $\mathrm{RC}$ ). A $2 \mathrm{~d} 3 \mathrm{v}$ (two-dimensional in coordinate and three-dimensional in velocity space) particle-in-cell-Monte Carlo model, including an external circuit, is developed to determine the role of the electron recapture in the discharge processes. The detailed discharge structure as a function of RC for two pressures (4 and 25 mtorr) is studied. The importance of electron recapture is clearly manifested, especially at low pressures. The results indicate that the discharge characteristics are dramatically changed with varying RC between 0 and 1 . Thus, the electron recapture at the cathode appears to be a significant mechanism in magnetron discharges and $\mathrm{RC}$ a very important parameter in their correct quantitative description that should be dealt with cautiously.
\end{abstract}

DOI: 10.1103/PhysRevE.72.056402

PACS number(s): 52.65.Rr, 52.65.Pp, 52.55.- s

\section{INTRODUCTION}

Magnetrons are a special type of processing glow discharges, which can work at low operating pressures. This is achieved by the use of a dc magnetic field at the cathode to confine the secondary electrons. Magnetrons have been intensively used for sputter deposition of different coatings for decades. Despite this fact, the understanding of all the processes and mechanisms occurring in these discharges is not yet complete. One of the sparsely touched problems in the literature is the electron return at the cathode and how exactly this phenomenon influences the discharge behavior. The magnetic confinement allows magnetrons to be operated at pressures of around a few millitorrs. Such low pressures lead to long mean free paths, thus allowing a significant number of electrons, ejected from the cathode, to return there during their first gyro period. Generally, the probability for this process depends on the initial energy of the electrons and on the number of collisions they suffer. Both elastic and inelastic collisions can prevent the electrons from returning to the cathode. The former can change the velocity vector of the electrons and so as to divert them to another magnetic field line, whereas the latter reduce their energy, so that they cannot overcome the sheath potential. The initial energy, at which the electrons leave the cathode, in most cases, plays a less important role. The reason is that the initial energy is normally less than the inelastic threshold of most of the gases used in magnetrons. As can be seen in [1], the energy distribution of the ejected electrons has a cutoff, given by the empirical relation $\varepsilon_{\text {cutoff }}=\Delta \varepsilon_{\text {ion }}-2 W$, where $\Delta \varepsilon_{\text {ion }}$ is the ionization potential of the feeding gas and $W$ is the work function of the cathode material. The maximum of the energy distribution is ca. $0.5 \varepsilon_{\text {cutoff. }}$. Therefore, the electron initial energy can change the probability for recapture only for the case of elastic collisions. This statement is valid for all kinds

*Electronic address: ivan.kolev@ua.ac.be of magnetrons. The specially shaped poloidal magnetic field in planar magnetrons, with magnetic lines of changing density and curvature and crossing the cathode, can create a magnetic mirror [2]. That mirror can cause a sharp turning of an electron shortly before its reaching the cathode, even though the electron may possess enough energy to strike the target. Taking into account the complexity of electric and magnetic fields in planar magnetrons, it is not possible to give a reasonably simple estimate of how much the magnetic mirroring can affect the electron recapture.

The effect of recapture of electrons at the target can interfere with the discharge characteristics in two ways. The more obvious one is that, if an electron is absorbed at the cathode without causing an electron impact ionization of a gas atom, it is as if it were not emitted at all. This claim is based on the fact that the discharge cannot be sustained without the ionizations caused by the cathode electrons. Therefore, on a macroscopic level, absorption of electrons at the cathode leads to an effective decrease of the secondary-electron emission coefficient (SEEC) $\gamma$, which gives the number of electrons produced by an ion striking the cathode. The SEEC is a very important parameter in the operation of dc magnetrons. Small variations of $\gamma$ cause significant changes in the amount of ionization, plasma density, and sheath architecture at fixed other parameters. The SEEC is energy dependent [3].

The second way the electron recapture influences the discharge is through the cathode potential. The more electrons are absorbed at the cathode, the lower the convective (conductive) discharge current is at the cathode and the more negative the cathode potential is. The latter will be illustrated further. This implies that electron recapture at the cathode may have a complex effect upon the discharge and a detailed investigation is necessary to describe it quantitatively. The results may be of interest to both simulators and experimentalists. In simulations, the reflection of electrons from the cathode surface is a boundary condition - in the fluid approach for the electron flux and density and in kinetic approach as a loss and birth process. In the existing simulation 
works on dc magnetrons the value of the reflection coefficient (RC) is frequently not explicitly given [4-6] or assumed to be zero $[7,8]$ without argumentation, meaning that all the electrons arriving at the cathode are considered absorbed. In a very detailed work on cylindrical magnetrons [9] the authors report using an $\mathrm{RC}$ of 0.5 and claim that little change in the discharge characteristics was found when varying the RC between 0.5 and 0.9 . Their choice of $R C=0.5$ is based on the early work of Thornton [10], where the value of 0.5 for cylindrical magnetrons is proposed without detailed justification. For planar magnetrons, only recently, a simple model was reported [11], devoted to the influence of the electron recapture on the discharge characteristics. The model is essentially a non-self-consistent trajectory calculation, with simplified collision processes, and an assumed one-dimensional (1d) electric field. Despite its simplicity, it shows clearly the importance of the electron recapture. Measurements and theoretical calculations $[12,13]$ of the reflection of low-energy electron beams from metal targets show that for energies up to $20 \mathrm{eV}$ (i.e., more than twice the maximal energy that an electron may have when returning at the cathode at typical operating conditions) the $\mathrm{RC}$ is never less than 0.1 (i.e., not equal to zero). Moreover, for energies between 0 and $5 \mathrm{eV}$ it grows very fast with decreasing energy, approaching a value of 0.55 [measured on $\mathrm{Cu}(001)$ ] and 0.8 (calculated) [13]. It is necessary to be mentioned that the electron interaction with the cathode in dc magnetrons involves electrons with energies under or around the work function of the cathode material. This means that there is no electron multiplication at the cathode, an effect known as a multipactor [14], which has been intensively studied for many years [15]. Moreover, there are a very limited number of true secondary electrons ejected from the cathode by electron impact. That is why the main mechanisms of interaction between the electrons returning at the cathode and the cathode surface are reflection (elastic or inelastic) and absorption $[13,14]$. A lot of attention has been devoted to the investigation of the interaction of electrons with metal surfaces and the probability for electron-induced secondary-electron emission. Interest in that subject has come mainly from areas such as scanning electron microscopy [16] and cathode emissions from vacuum tubes. However, the characteristic energies of the primary electrons there are from a few hundred $\mathrm{eV}$ to a few tens of $\mathrm{keV}$, while as has been mentioned above, the energies of interest in the dc sputtering magnetrons do not exceed a few eV. A relatively recent work to model electron interaction with metal surfaces with improved accuracy for the low-energy interval has been reported by Furman and Pivi $[17,18]$. It will be discussed in more detail in Sec. III C and Sec. IV G.

The above discussion motivates a detailed investigation of the electron recapture at the cathode in dc magnetrons. The purpose of the present article is to perform such a survey by means of a self-consistent numerical simulation in order to examine the existence and influence of the electron recapture at the cathode and to compare different ways of inclusion of this effect in numerical simulations.

\section{DESCRIPTION OF THE MAGNETRON}

The scheme of the planar magnetron used in the present simulation is shown in Fig. 1. An axisymetric magnetic field

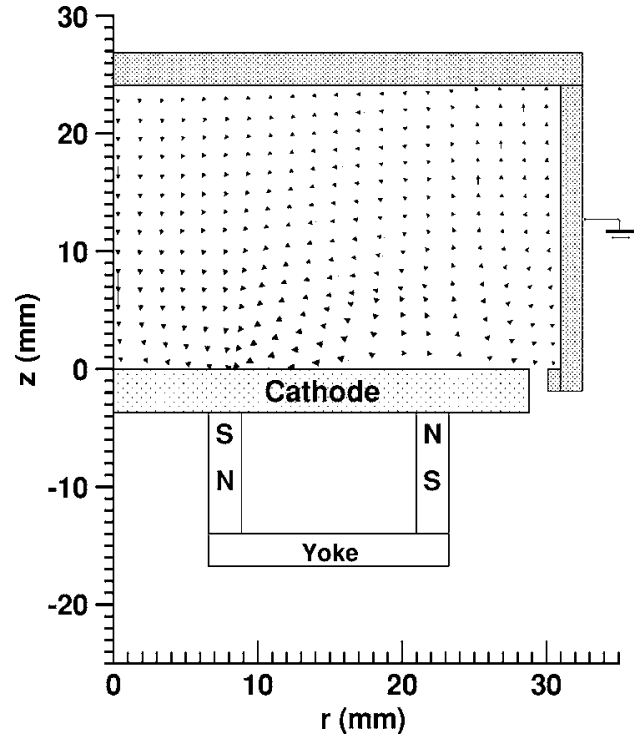

FIG. 1. Scheme of the magnetron in the study with the magnetic field. The maximum strength of the magnetic field is $300 \mathrm{G}$. The scheme is cylindrically symmetrical towards the axis $r=0$.

is created by two concentric magnets located under the powered electrode - the cathode. The magnetron is balanced, which means that the majority of the magnetic flux lines originate at and return to the cathode surface without crossing the anode. All walls, except the cathode, are grounded and act as an anode. The smallest separation between the electrodes is equal to $2 \mathrm{~mm}$, and the distance between the cathode and the opposite anode plate is $24 \mathrm{~mm}$. The cathode is a copper disk with a thickness of $3 \mathrm{~mm}$ and a diameter of $58 \mathrm{~mm}$. The discharge is maintained by a dc power supply, which can be run in a constant current or in a constant voltage mode.

\section{SIMULATION MODEL}

The model used in the present work is based on the particle-in-cell-Monte Carlo collision (PIC-MCC) method. The outlines of this technique are given in detail in [19] and [20]. The overall idea is that a limited ensemble of charged particles, commonly referred to as superparticles (SP's), represents the entire discharge. Thus, each SP replaces $W$ real particles (i.e., electrons and ions), where $W$ is known as weight and all SP's are consecutively traced. Their velocities and coordinates are obtained as numerical solutions of their equations of motion in a force field created by the applied magnetic field, the electric field created by the powered electrode, and the electric field generated by the SP'sthemselves. The magnetic field is assumed strong enough to be undisturbed by the spatial distribution of the charge and so is constant. In other words, the magnetic field formed by the SP current is much weaker than the external one. The electric field is obtained through a numerical solution of Poisson's equation with a source term given by the SP charge density. The SP's are allowed to collide with the parent gas atoms and to interact with the electrodes. Coulomb interactions are disregarded, since the degree of ionization is less 
than $0.13 \%$. The sputtered atoms from the cathode are not included in the model. The background gas ( $\mathrm{Ar}$ ) is assumed to be at room temperature $(300 \mathrm{~K})$ and to have a constant density. Owing to the axisymmetrical magnetic field and the cylindrical symmetry of the discharge vessel, the whole discharge is assumed cylindrically symmetrical. Such claim has been justified for a similar system in [5], where the authors, performing a fully $3 \mathrm{D}$ simulation, have established the absence of angular dependence of the discharge parameters. Thus, the present simulation is performed in cylindrical $(r, z)$ coordinate space. However, all three velocity components are taken into account. This is necessary in order to satisfy energy conservation. The simulation is carried out until all major discharge parameters converge to steady-state values.

\section{A. Initial conditions and equation of motion}

The simulation starts with some initial state of the system - a given velocity and density distribution of the initial number of SP's, usually a homogeneous electron $\left(n_{e}\right)$ and ion $\left(n_{i}\right)$ density, and a Maxwellian velocity distribution. The initial densities in the present simulation are $n_{e}=n_{i}=1$ $\times 10^{15} \mathrm{~m}^{-3}$ and the SP initial number is 500000 . For the velocity distribution the ions are assumed to have a temperature $T_{i}=0.026 \mathrm{eV}$ and the electrons $T_{e}=1 \mathrm{eV}$. Alternatively, the simulation could be initiated with converged results from a previous run. Between two consecutive collisions, each SP moves according to its equation of motion

$$
m \frac{d \boldsymbol{\nu}}{d t}=q(\boldsymbol{E}+\boldsymbol{\nu} \times \boldsymbol{B}),
$$

where $m$ is the mass and $q$ is the charge of the superparticle, $\boldsymbol{\nu}$ is its velocity, $\mathbf{E}=E\left(E_{r}, 0, E_{z}\right)$ is the electric field, and $\boldsymbol{B}$ $=B\left(B_{r}, 0, B_{z}\right)$ is the magnetic field. It is integrated using the standard time-centered explicit integration scheme called "leapfrog" [20]. The rotation $(\boldsymbol{\nu} \times \boldsymbol{B})$ term is treated according to the algorithm suggested by Boris [21]. If $\boldsymbol{x}^{n}$ are the known coordinates of all SP's at the moment $n \Delta t$ and $\boldsymbol{\nu}^{n-1 / 2}$ are the known velocities at the moment $(n-1 / 2) \Delta t$, then $\rho^{n}(\boldsymbol{x})$, the charge density, is also known and, hence, the electric field $\boldsymbol{E}^{n}(\boldsymbol{x})$ as a solution of Poisson's equation. To alleviate the effect of the numerical charge density fluctuations, which is a direct result of using a reduced ensemble of SP's, digital filtering is used [22]. This means that the charge density sampled on the grid from the SP positions is smoothed before being used as a source term to Poisson's equation. The principle is that each grid value is replaced by a new one, obtained by the rough neighboring values.

The next step is to determine $\boldsymbol{\nu}^{n+1 / 2}$ from the already known quantities $\boldsymbol{\nu}^{n-1 / 2}, \boldsymbol{E}^{n}(\boldsymbol{x})$, and $\boldsymbol{B}^{n}(\boldsymbol{x})$. Finally, the new coordinates $\boldsymbol{x}^{n+1}$ are computed using $\boldsymbol{\nu}^{n+1 / 2}$ and $\boldsymbol{x}^{n}$. The probability of a collision is examined at the middle of the time step $\Delta t$, where the energy is known. In the case of a collision, the particle velocity $\boldsymbol{\nu}^{n+1 / 2}$ is replaced by the post-collision velocity $\boldsymbol{\nu}^{* n+1 / 2}$. A question arises how to advance a SP in a case of velocity jump. An obvious solution is to halve the interval $\Delta t$. In the first subinterval the collided SP is moved from $\boldsymbol{x}^{n}$ to $\boldsymbol{x}^{n+1 / 2}$ using $\boldsymbol{\nu}^{n+1 / 2}$, then, in the second, from $\boldsymbol{x}^{n+1 / 2}$ to $\boldsymbol{x}^{n+1}$ using $\boldsymbol{\nu}^{* n+1 / 2}$. But the leapfrog algorithm requires shifting half a time step between known values of $\boldsymbol{x}$ and $\boldsymbol{\nu}$. To solve that problem, a forward, instead of a centered, integration method must be implemented with the same order of accuracy. Such a method can be the fourth-order RungeKutta, for instance. However, using it will lead to an unwanted intensification of the computation. Instead, the authors have chosen to apply the leapfrog scheme, even though the time centering has been violated. The comparison between the two approaches shows that there is practically no difference in the overall behavior of the simulation, while the computational benefits of the second approach are obvious. Additional time saving is achieved by implementing the socalled "null-collision" technique [23]. The advantage of the method is the possibility to determine the maximum number of SP's, which can suffer any kind of collision in a given time step. Then, only this number of SP's, randomly selected among all the SP's, is checked for collisions. Since at low pressures only few collisions happen per time step, while the size of the ensemble is usually in the order of $10^{5}$ SP's, a considerable reduction in computational time is attained. The method is unconditionally stable for calculating swarm parameters, but was questioned by Nanbu [24] for applications in magnetron simulations due to the strongly inhomogeneous electrical field. Our comparison between the null-collision approach and the technique proposed by Nanbu [25] shows that both methods yield identical results for the operating conditions under consideration. For that reason, the nullcollision method is used here. To keep the computational intensity under control, an upper limit of SP's, $N_{\max }$, is set. Each time the number of ion SP's exceeds $N_{\max }$, the number of all SP's is reduced twice. Correspondingly, their weight $W$ is doubled. The SP's to be removed are randomly selected.

\section{B. Grid and Poisson's equation}

The electrical potential $V$ in the system follows from Poisson's equation, which in cylindrical $(r, z)$ coordinate system reads

$$
\frac{1}{r} \frac{\partial}{\partial r} r \frac{\partial V}{d r}+\frac{\partial}{\partial z} \frac{\partial V}{\partial z}=-\frac{q}{\varepsilon_{0}}\left(n_{i}-n_{e}\right),
$$

where $\varepsilon_{0}$ is the dielectric permittivity of free space and $q$ is the elementary charge. Equation (1) is an elliptic partial differential equation with separable coefficients. This favors the use of some of the so-called rapid elliptic solvers [26], which are much more efficient than the more frequently used meshrelaxation methods (MRM's) [26]. Since there is no periodicity in either of the directions, fast Fourier transform techniques are inapplicable. Instead, the cyclic reduction method (CRM) can be employed. The description of the general CRM is given [27]. If an equidistant grid in the $z$ direction is used, the CRM algorithm is even faster. An additional advantage of the CRM is that as a direct method, it is presumably more accurate than any of the MRM's, which are iterative.

By using the superposition principle, $V$ could be presented as $V=V_{P}+U_{0} V_{L}$, where $V_{P}$ is the potential created by the space charge in the discharge, $V_{L}$ is the potential due to applied voltage with magnitude $1 \mathrm{~V}$, and $U_{0}$ is the cathode 
voltage [28]. Then $V_{P}$ can be found as a solution of Eq. (1) with the Dirichlet boundary condition $V_{P}=0$ at the surfaces $z=0, z=z_{\max }$, and $r=r_{\max }$. Since there is no linear charge at the origin $r=0$, the only physical boundary condition there could be is

$$
\left.\frac{\partial V_{P}}{\partial r}\right|_{r=0}=0 .
$$

The potential $V_{L}$ is a solution of the Laplace equation

$$
\Delta V_{L}=0,
$$

with boundary conditions $V_{L}=0$ at the grounded electrodes and $V_{L}=1$ at the cathode. In the gap between the cathode and anode at $z=0, V_{L}$ is assumed linearly decaying from 1 to 0 with the distance from the cathode edge. At $r=0$ again Eq. (2) is imposed with $V_{L}$, instead of $V_{P}$. Equation (3) needs to be solved only once at the beginning of the simulation. Equations (2) and (3) and are discretized using a standard fivepoint stencil and solved on a grid with $\Delta z=0.01 \mathrm{~mm}$ and $\Delta r=0.33 \mathrm{~mm}$. The grid size is chosen so that $\Delta z$ is less than the Debye length, which is about $0.15 \mathrm{~mm}$ for the present conditions. Once $V$ is found, the electrical field $\boldsymbol{E}$ is obtained from

$$
\boldsymbol{E}=-\operatorname{grad} V
$$

using central finite differences over two grid cells.

\section{Collisions and post-collision velocities}

Only collisions between electrons or ions and argon atoms are considered. For all kinds of collisions the probability $P_{k}$ is given by

$$
P_{k}=1-\exp \left[N_{A r} \sigma_{k}\left(\varepsilon_{i}\right) \sqrt{2 \varepsilon_{i} / m_{i}} \Delta t\right],
$$

where $k$ is the type of collision, $\sigma_{k}$ is the cross section for the $k$ th type of collision, $\varepsilon_{i}$ is the kinetic energy of the incident particle, $m_{i}$ is its mass, and $N_{A r}$ is the Ar density. The collisions for the electrons include (i) elastic scattering from the argon atoms, (ii) excitation, and (iii) single ionization of argon atoms. To simplify the model all different excitation processes are combined into a single process with a threshold of $11.6 \mathrm{eV}$. In case of (i) the total integrated elastic cross section is needed and not the elastic momentum transfer cross section. Cross section data for the above processes are adopted as follows: for (i) and (ii) tabulated data of Hayashi [29] and for (iii) an analytical fit by Phelps and Petrovich [3]. The whole set is shown in Fig. 2. The scattering is considered anisotropic. The scattering angle $\chi$ in a c.m. system, as a function of $\varepsilon_{i}$, is calculated, based on an assumption for screened Coulomb potential, according to [30]

$$
\chi=\arccos \left(1-\frac{2 R N}{1+8 E(1-R N)}\right),
$$

where $R N \in[0,1]$ is a uniformly distributed random number, $E=\varepsilon_{i} / E_{0}$, and $E_{0}$ is the atomic unit of energy $\left(E_{0}\right.$ $=27.21 \mathrm{eV}$ ). In the case of ionization the energy of the ejected electron, $\varepsilon_{e j}$, is given by [31]

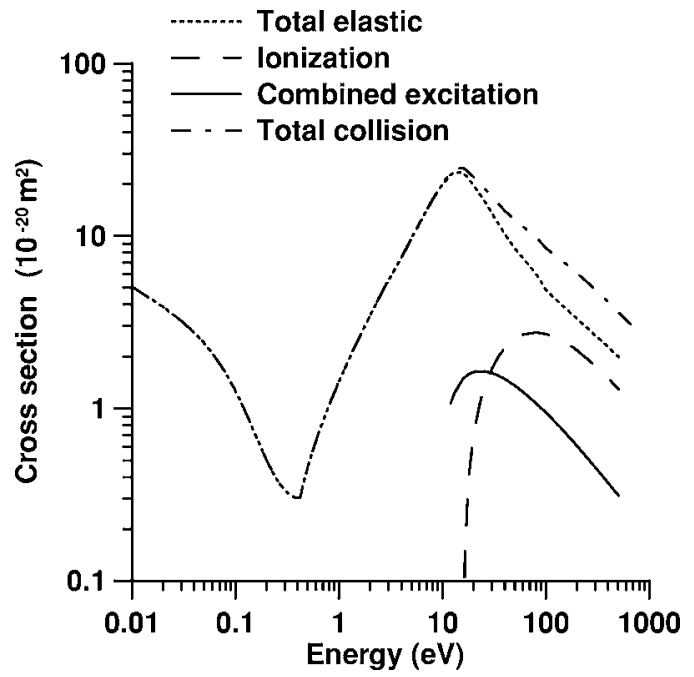

FIG. 2. Cross sections for electron-Ar collisions.

$$
\varepsilon_{e j}=D\left(\varepsilon^{\prime}\right) \tan \left(\frac{\varepsilon^{\prime}-\Delta \varepsilon_{i o n}}{2 D\left(\varepsilon^{\prime}\right)}\right),
$$

where $D$ is a weakly variable parameter, taken equal to 10 for $\mathrm{Ar}, \varepsilon^{\prime}$ is the precollision energy of the incident electron, and $\Delta \varepsilon_{i o n}$ is the ionization potential of Ar. The post-collision energy of the incident electron is then $\varepsilon_{i}=\varepsilon^{\prime}-\varepsilon_{e j}-\Delta \varepsilon_{i o n}$. When excitation happens, $\varepsilon_{i}=\varepsilon^{\prime}-\Delta \varepsilon_{e x c}, \Delta \varepsilon_{e x c}$ being the excitation threshold energy. The subtraction of the energy in all of the above-mentioned situations is implemented via reducing the speed of the electrons, but without changing the ratio between velocity components. Since only the magnitude of the newborn electron velocity is known, its direction is assumed to be parallel with the precollision velocity of the ionizing electron. In all of the inelastic collisions, after the velocity jump is realized, the electrons are scattered according to Eq. (4). The new post-collision velocities as a function of $\chi$ are calculated according to [32].

Ions participate in a symmetric ion-atom scattering. This scattering can be considered as consisting of two components [33]: isotropic and a well-expressed backward component, which at high energies coincides with symmetric charge transfer, but at low energies its magnitude differs from that of the symmetric charge transfer as calculated from quantum mechanics considerations [34]. There is a lack of separate cross-section data for elastic scattering and symmetric charge transfer in the literature. In [33], Phelps gives analytical expressions for the isotropic and backwards components of the symmetric ion-atom scattering. In the present model, the authors use these expressions to treat the ion collisions.

The post-collision velocities are determined as follows: in the case of isotropic scattering again according to [32] with $\chi$ determined from $\cos \chi=\mathrm{RN} 1$, where $\mathrm{RN} 1 \in[-1,1]$ is a uniformly distributed random number. In the case of backward scattering the ion obtains the atom velocity, determined from sampling a Maxwellian distribution of a temperature of $300 \mathrm{~K}$. In all the collision processes in the model the azimuthal scattering angle in a c.m. system is considered equal 
to $2 \pi \times \mathrm{RN}$. All random numbers mentioned above are independent.

When reaching the electrodes, the ions are considered unconditionally absorbed. Depending on their energy, a secondary electron can be released. The SEEC is calculated using the formula proposed by Phelps and Petrovich [3] and compared to a $\mathrm{RN}$. If $\mathrm{RN} \leqslant \mathrm{SEEC}$, a secondary-electron emission takes place. The electrons, which return at the cathode, are either reflected or absorbed. Two different approaches are used to determine which process occurs. The first one is based on the assumption of a constant predefined RC. Each time an electron strikes the cathode the RC is compared to a $\mathrm{RN}$. If $\mathrm{RN} \leqslant \mathrm{RC}$, reflection happens; otherwise, the electron is absorbed at the cathode. The use of a constant $\mathrm{RC}$ allows us to examine numerically how sensitive the discharge is to different values of the $\mathrm{RC}$ or, in other words, how important the electron recapture at the cathode is. The advantage of such a procedure is its simplicity and the possibility to have a fitting parameter when numerical data are compared to experimental ones. Of course, the process of the interaction of an incident electron with a metal surface is probabilistic and generally speaking quantum mechanical. It is also dependent on the electron energy and incidence angle. In the case of magnetrons, the electrons arriving back at the cathode are relatively monoenergetic; thus, it may be expected that using a constant RC still may produce correct results. This claim will be checked later on.

The second approach for dealing with returning electrons on the cathode is based on the model proposed by Furman and Pivi $[17,18]$ It is a microscopic probabilistic model, designed to give the probability $P_{n}\left(E_{0}, \theta_{0}\right)$ for emission of $n$ $=0, \ldots, \infty$ electrons from a metal surface in the case of a single electron-surface interaction-i.e., an electron with kinetic energy $E_{0}$ striking a surface at an angle $\theta_{0}$. The case $n=0$ represents the absorption of the incident electron, $n=1$ its reflection (elastic or not), and $n \geqslant 2$ true secondary electrons. Furthermore, the model gives the initial velocity of the ejected electrons. Although it has been developed to be included in simulations of electron-cloud effect [35,36], where $E_{0}$ is normally much higher, it is valid for the energies of interest in the present study.

At the walls all electrons are considered absorbed. This is motivated by speeding up the procedure. It is not a severe restriction, because a reflected electron from a wall quickly returns there due to the direction of the electric field, with an insignificant probability to produce an inelastic event.

\section{External circuit}

Being glow discharges, magnetrons have negative differential resistance and in the absence of a current limiting device their regime of operation is not defined. From a modeler's point of view, this means that the simulation may converge, if at all, to a current value belonging to a different region of the volt-ampere characteristic. To overcome the problem an external circuit must be incorporated selfconsistently to the discharge, thus allowing the cathode potential to be calculated as a function of both the circuit parameters and discharge processes. In this simulation a simple circuit, consisting of a constant-voltage source and a ballast resistor in series with the discharge, is employed. The coupling between the circuit and discharge is maintained via satisfying charge conservation at the cathode,

$$
A \frac{d \sigma}{d t}=I_{e x t}(t)+Q_{d i s c h},
$$

where $\sigma$ is the total surface charge at the cathode, $I_{e x t}$ is the external circuit current, $A$ is the cathode surface, and $Q_{\text {disch }}$ is the charge deposited from the discharge on the cathode during a period $d t$. The total surface charge can be determined independently of Eq. (5) applying Gauss's theorem on a box surrounding the cathode. Then according to Kirchhoff's voltage loop law, the cathode potential $U_{0}=V_{e x t}-R_{e x t} I_{e x t}$. The overall principles of simultaneous circuit and discharge simulation are given in [19]. A detailed numerical procedure for the case of $(x, y)$ Cartesian geometry is proposed in [37]. In the present paper, the same procedure as in [37] is followed, after the necessary modifications to account for the cylindrical $(r, z)$ geometry.

\section{E. Time step, stability, and convergence}

The time step is limited by the stability criterion of the leapfrog algorithm. For Maxwellian plasma it reads [19]

$$
\omega_{c h} \Delta t \leqslant 1.62,
$$

where $\omega_{c h}$ is the characteristic frequency in the simulated system. The above criterion has been deduced using a harmonic oscillator model; thus, $\omega_{c h}$ is an angular and not a linear frequency. In magnetrons, there are two frequencies which must be dealt with: the electron plasma frequency

$$
\omega_{p e}=\sqrt{n_{e} q_{e}{ }^{2} / \varepsilon_{0} m_{e}}
$$

and the electron gyrofrequency

$$
\omega_{g e}=q_{e} B / m_{e},
$$

where $m_{e}$ and $q_{e}$ are the mass and charge of the electron. Hence, $\omega_{c h}=\max \left\{\omega_{p e}, \omega_{g e}\right\}$. In the present simulation $B$ does not exceed $300 \mathrm{G}$, so $\omega_{g e} \leqslant 5.28 \times 10^{9} \mathrm{rad} / \mathrm{s}$. Here $\omega_{p e} \mathrm{de}-$ pends on $n_{e}$, which is not known in advance and can vary largely during the simulation.

Therefore, every 2000 time steps the maximum $n_{e}$ is determined and $\Delta t$ is adjusted to satisfy Eq. (6). At these moments time centering is lost, but no instabilities have been observed. Another very important restriction is the Courant criterion

$$
\nu \Delta t / \Delta z \leqslant 1,
$$

where $\nu$ is some mean characteristic velocity. The meaning is that, if Eq. (7) is violated, too many SP's are jumping over field variations, which leads to numerical heating. In equilibrium plasmas $v$ can be replaced by thermal velocity. Magnetrons are nonequilibrium plasma devices. The discharge exists due to the presence of highly energetic nonequilibrium electrons emitted from the cathode. If $\Delta t$ is fixed to obey Eq. (7) for their typical velocities, this will result in very long computational times. Since these fast electrons are only a 


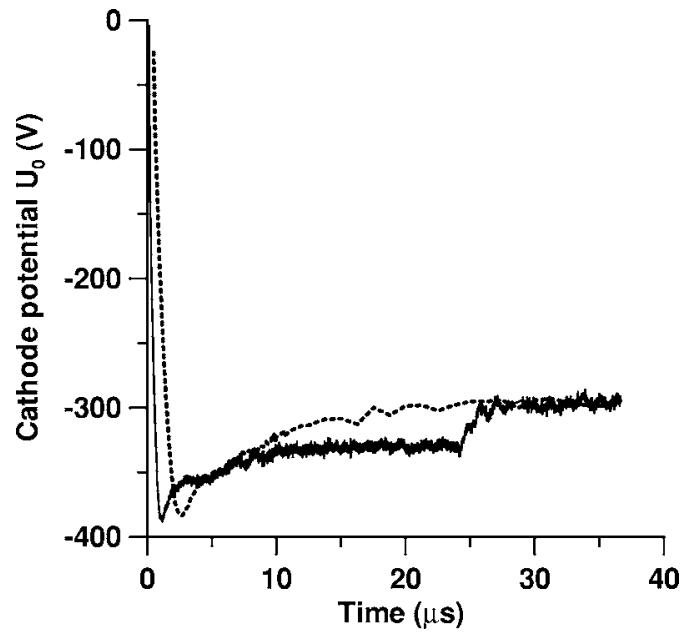

FIG. 3. Convergence of the cathode potential $U_{0}$ for $p$ $=4$ mtorr and $\mathrm{RC}=0.5$. The dashed line represents a simulation initiated with a homogeneous plasma density. The solid line corresponds to a simulation started with the converged data of a run with $\mathrm{RC}=0$. The transition occurs at $t=24.2 \mu \mathrm{s}$.

tiny fraction of the whole electron population, $\Delta t$ is set to obey Eq. (6) only. The Courant criterion is fulfilled by checking the path $\Delta s$ traveled by every electron per time step. If $\Delta s>\Delta z$, the electron is returned to its initial position and then moved twice with the $\frac{1}{2} \Delta t$ in a manner preserving time centering. The procedure is iteratively repeated until $\Delta s$ becomes smaller than $\Delta z$. Acting that way is computationally much more efficient than setting $\Delta t$ to obey Eq. (7) for all electron velocities. Thus, $\Delta t$ varies during the simulation be- tween, approximately, $10^{-10}$ and $10^{-12} \mathrm{~s}$. Taking into account the relative slowness of the ions in comparison to the electrons, the ions are moved only once per ten time steps, a procedure known as electron subcycling [38].

Normal convergence times have been found to be not less than $20 \mu \mathrm{s}$. When convergence is reached, the simulation is restarted with the double-SP number for reducing the noise and so improving the statistics.

\section{RESULTS AND DISCUSSION}

First, a simulation initiated with homogeneous density, $\mathrm{RC}=0$ and $p=4$ mtorr, has been brought to convergence. Then the results of this run have been used as initial conditions in all following runs, where $\mathrm{RC}$ and/or $p$ have been varied. For all cases the external circuit parameters are $V_{\text {ext }}$ $=450 \mathrm{~V}$ and $R_{\text {ext }}=1 \mathrm{k} \Omega$. For $p=4$ mtorr, runs with $\mathrm{RC}=0$, $0.25,0.5,0.75,0.85$, and 1 have been done and for $p$ $=25$ mtorr with $\mathrm{RC}=0,0.5$, and 1 . The independence of the steady-state values from the initial conditions applied in the model is illustrated in Fig. 3, where convergence of the cathode potential $U_{0}$ to a steady-state value is shown. The jump of the solid curve at $t$ approximately equal to $25 \mu$ s corresponds to the moment when a converged simulation with $\mathrm{RC}=0$ has been restarted with $\mathrm{RC}=0.5$.

Second, a simulation at $p=4$ mtorr with recapturing treated, following the model of Furman and Pivi [17,18], has been performed, from which an averaged value of the RC has been obtained. Then a simulation with that $\mathrm{RC}$ has been done.
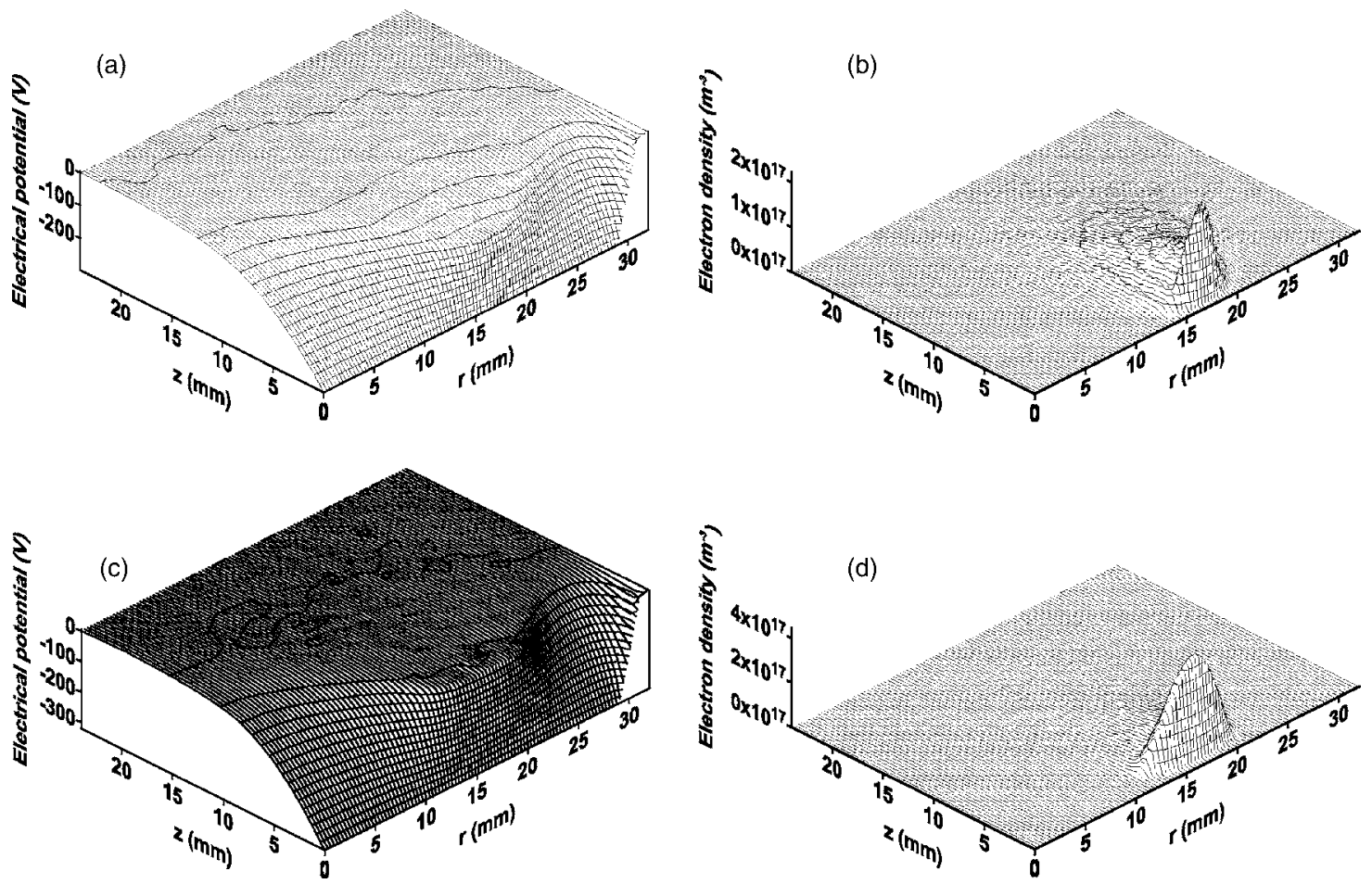

FIG. 4. Discharge characteristics: (a) electric potential at $p=4$ mtorr, (b) electron density at $p=4$ mtorr, (c) electric potential at $p$ $=25$ mtorr, and (d) electron density at $p=25$ mtorr. 


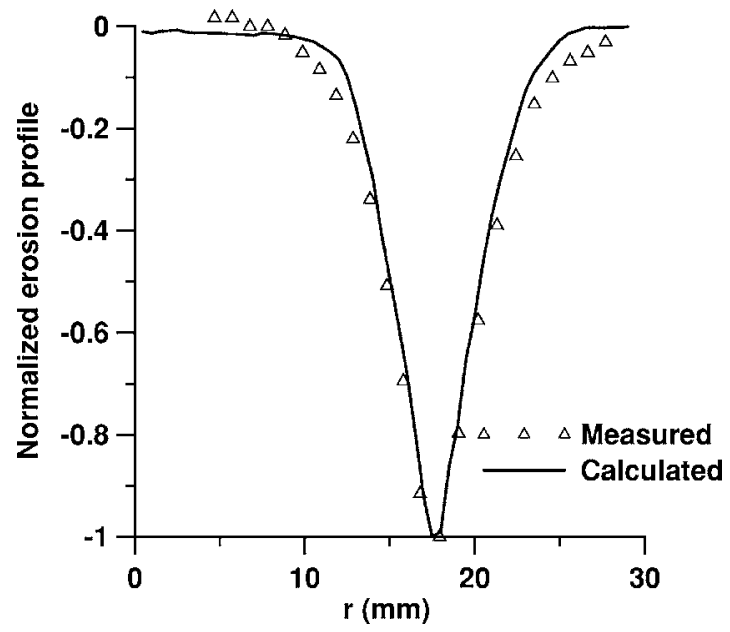

FIG. 5. Erosion profile at $p=4$ mtorr and $\mathrm{RC}=0.5$. The solid line represents the calculated results and open triangles the measured data.

\section{A. Overall view}

The overall picture of the discharge is represented in Fig. 4 , where the potential and electron density for $\mathrm{RC}=0.5$ are shown for a pressure of $p=4$ and 25 mtorr. The potential has clearly expressed radial dependence. At both pressures the plasma is confined into a narrow region, centered at about $r=17.2 \mathrm{~mm}$, where $B_{r}$ has a maximum. At the lower pressure the plasma density is lower. The electrons do not suffer enough collisions, which results in the fact that their mobility is significantly reduced, leading to the formation of a region where the space charge is negative. A presheath with significant electric field is formed. The electrons that escape the magnetic trap immediately before the cathode can accelerate in this presheath and produce ionization farther from the cathode. This explains the fact that the electron density in the region $z>5 \mathrm{~mm}$ is higher at $p=4$ mtorr [Fig. 4(b)] than at $p=25$ mtorr [Fig. 4(d)].

The formation of a negative space-charge region [Fig. $4(\mathrm{a})]$ is in principal agreement with the 1D PIC-MCC simulation of a cylindrical post-magnetron [9]. The calculated potential distribution is also in very close correspondence to the measured one by Bradley et al. [39]. In their investigation of a planar magnetron operated at a pressure of 2 mtorr and a cathode voltage of $-330 \mathrm{~V}$ the plasma potential is entirely negative in the whole region of measurement, being around $-40 \mathrm{~V}$ at $1 \mathrm{~cm}$ from the cathode. At the same location, the calculated plasma potential is $-38 \mathrm{~V}$ [Fig. 4(a)] at $p=4$ mtorr mtorr and $V_{c}=-295 \mathrm{~V}$.

The electron density presented in Figs. 4(b) and 4(d) reproduces satisfactorily the experimental data obtained by Langmuir probe measurements. For example, the calculated peak value $p=4$ mtorr is $2 \times 10^{17} \mathrm{~m}^{-3}$, while in Ref. [39] it is $9 \times 10^{16} \mathrm{~m}^{-3}$ at $p=2$ mtorr. The plasma decay with the distance from the cathode, as well as the radial variation of the electron density, agrees very well with the data reported in Ref. [40] for $p=5$ mtorr.

To check the results of the model the erosion profile for the case of $p=4$ mtorr and $\mathrm{RC}=0.5$ is calculated from the steady-state data for the ion energy distribution at the cathode [41] and compared to the experimentally measured data (Fig. 5). The latter were obtained after sputtering for $4 \mathrm{~h}$ $30 \mathrm{~min}$ at $p=4$ mtorr [42]. The calculated profile is in a very good agreement with the measured one: both the maxima and shapes of the calculated and measured craters coincide. The calculated crater is everywhere shallower. The difference is around $7 \%$ in the central part of the crater and increases at the periphery. This difference can be related to the contribution of the fast neutrals (not included in the model) to the sputtering process. Being less directional than ions, their relative contribution is expected to increase with the distance measured from the center of the crater.

\section{B. Electron trajectories}

To visualize the effect of the $\mathrm{RC}$ on the discharge on a microlevel, a sample electron, ejected from the cathode, has been traced for the cases of $\mathrm{RC}=0$ [Fig. 6(a)] and $\mathrm{RC}=1$ [Fig. 6(b)]. For $\mathrm{RC}=0$ the electron returns at the cathode without suffering any collisions, which means its existence remains unseen by the discharge, since no ionization has happened and thus no contribution to the discharge maintenance has occurred. Of course, not all of the electrons, ejected from the cathode, exhibit the same behavior. Some of them manage to collide during this first gyro orbit. A typical particularity of the electron motion in complex electric and magnetic fields can be seen at the turning point at $r$ $\approx 17.6 \mathrm{~mm}$. At that moment the electron possesses enough
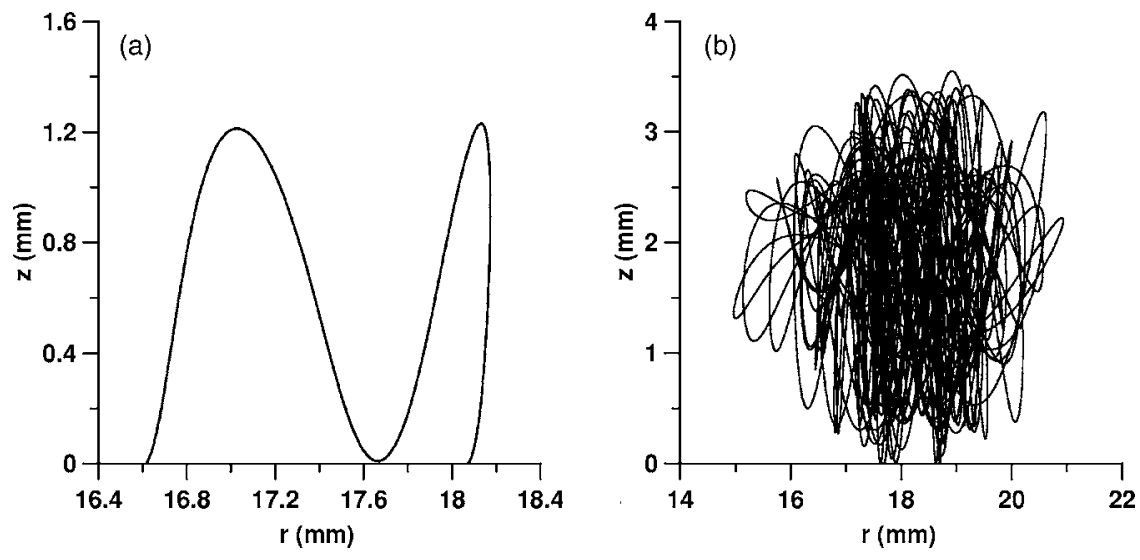

FIG. 6. Trajectory of a sample electron emitted from the cathode for (a) $\mathrm{RC}=0$ and (b) $\mathrm{RC}=1$. 


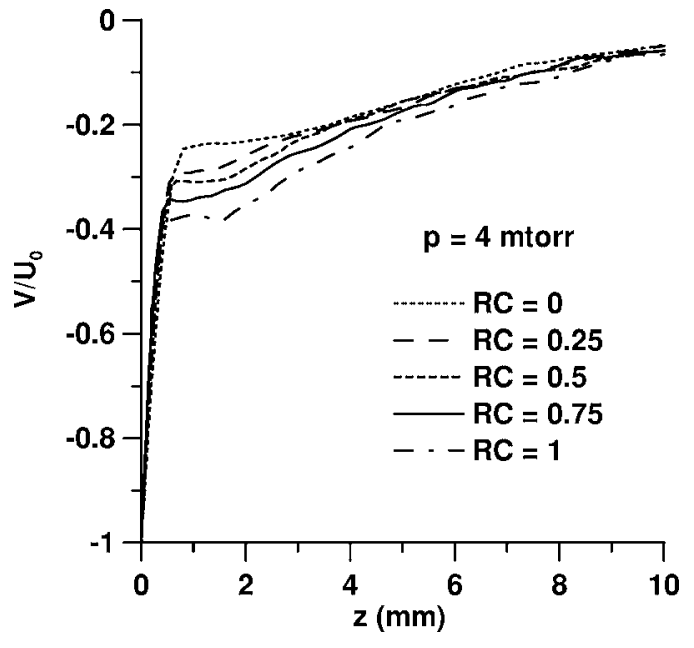

FIG. 7. Plasma potential $V$, normalized with respect to the cathode potential $U_{0}$, along $r=17.2 \mathrm{~mm}$ at $p=4$ mtorr for different values of the reflection coefficient.

energy to overcome the sheath potential, but nonetheless, it is turned back just immediately before the cathode.

A rather different picture can be seen in Fig. 6(b). Being allowed a full reflection, the sample electron stays in the discharge for significantly longer time and eventually manages to ionize an argon atom. In fact, setting $\mathrm{RC}=1$ leads to almost $100 \%$ probability that an electron, released from the cathode, will produce ionization. This is so, because the electron needs many elastic collisions to escape the magnetic trap, so the path traveled during its lifetime becomes longer than the mean ionization path.

\section{Sheath architecture}

More reflection means more electrons in front of the cathode and more negative space charge there. An illustration of this statement is shown in Fig. 7, where the potentials at $r$ $=17.2 \mathrm{~mm}$ (above the "race track"), normalized with respect to the cathode voltage, for all $\mathrm{RC}$ values are plotted at $p$ $=4 \mathrm{mtorr}$. The bigger the RC, the steeper the cathode fall and the more negative the space charge in the vicinity of the cathode. The latter leads to lower bombarding energies at the cathode, since the maximum of the ionization is situated at around $1.2 \mathrm{~mm}$ from the cathode. Thus, the majority of the ions cannot gain the entire discharge potential difference.

\section{Ionization rate and density}

The steepness of the potential results in a stronger electric field, which creates a possibility for a denser population at the high-energy tail of the electron energy probability function (Fig. 8). All this inevitably causes an increased amount of ionization and higher electron density, as shown in Figs. 9 and 10 for $p=4$ mtorr. Except in the sheath, the ion density profile (not shown here) is identical to the electron density, because the ions are electrostatically bound to the electrons.

The ionization rate for $p=25$ mtorr as a function of $\mathrm{RC}$ is presented in Fig. 11. The same tendency as at $p=4 \mathrm{mtorr}$ appears, but it is weaker, as might be expected. The ampli-

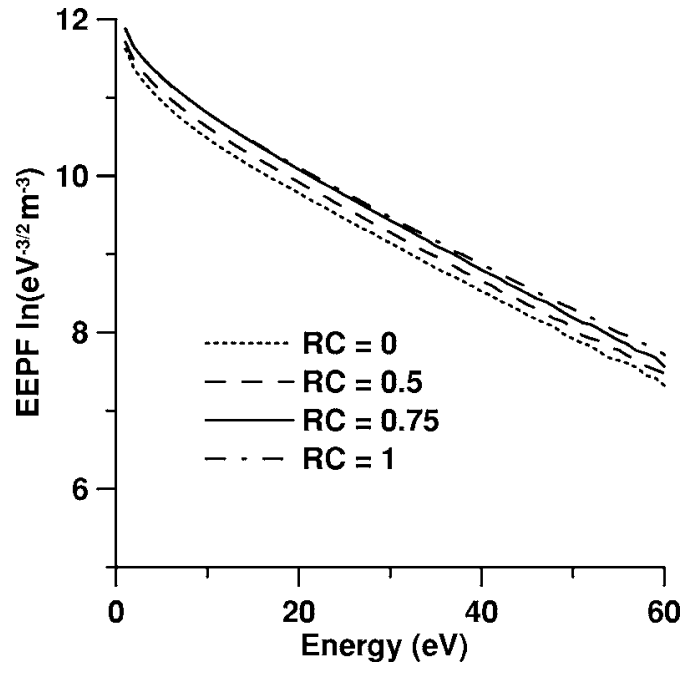

FIG. 8. Electron energy probability function at $p=4$ mtorr as a function of the reflection coefficient.

fication factor, defined as the ratio of the maximum ionization rate at $\mathrm{RC}=1$ over the value at $\mathrm{RC}=0$, is 2.67 at the lower pressure, while at the higher pressure it is 1.67.

\section{E. Cathode potential and discharge current}

The authors' intention has been to keep either $U_{0}$ or $I_{\text {ext }}$ constant, while changing the RC. This has proved to be not possible. The reason is that the influence of the RC is so strong that there is no single value of either $U_{0}$ or $I_{e x t}$ that can sustain the discharge throughout the whole range of $\mathrm{RC}$ values. If the converged $U_{0}$ for $\mathrm{RC}=0$, for example, is tried to be kept for higher RC, the discharge is overpumped and the simulation moves towards arc, becomes unstable, and eventually terminates. The instability is caused by a growth of the amount of ionization, strong enough to form a significant local space-charge perturbation.

With an external circuit of the type used here, there are two ways to maintain constant discharge voltage. Either the external voltage has to be increased or the ballast resistance

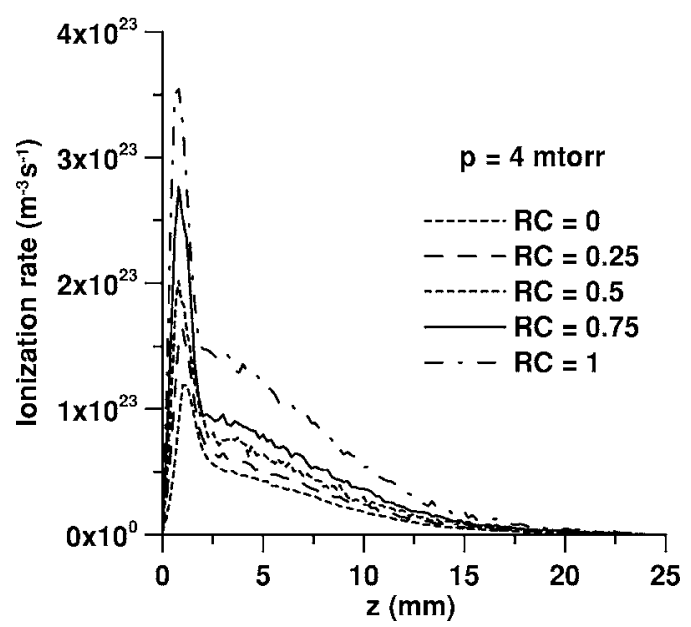

FIG. 9. Ionization rate along $r=17.2 \mathrm{~mm}$ at $p=4$ mtorr as a function of the reflection coefficient. 


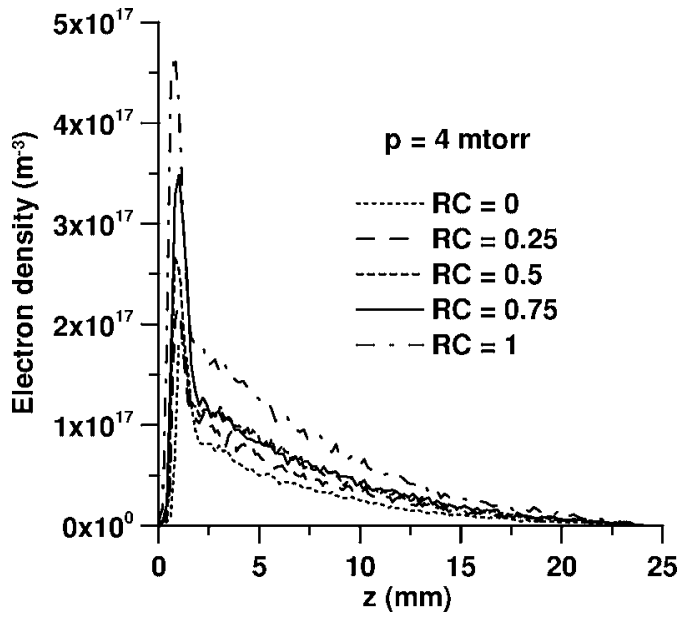

FIG. 10. Electron density along $r=17.2 \mathrm{~mm}$ at $p=4$ mtorr as a function of the reflection coefficient.

ought to be decreased, provided the discharge voltage has dropped and needs to be returned at its previous level. However, either of the two ways is essentially pumping more energy in the discharge, hence more ionization. More ionization means again lower cathode voltage due to an increased positive charge deposition, which again calls for further power from the source.

The opposite situation happens when $U_{0}$, obtained for $\mathrm{RC}=1$, is attempted to be kept for lower RC. Then the discharge just extinguishes, because $U_{0}$ is too low to maintain the discharge at low reflection rates. The process is gradual and passes through a condition close to a Townsend discharge-stable, but with extremely weak current. For this reason, all present simulations are performed with constant external parameters, allowing the discharge to achieve a steady-state voltage and current. Their dependences on RC are shown in Figs. 12 and 13, respectively. The discharge current increases with the increase of $\mathrm{RC}$, while the discharge voltage drops. This simulation result is directly connected to the well-known fact that glow discharges have negative differential resistance. It can be easily explained by

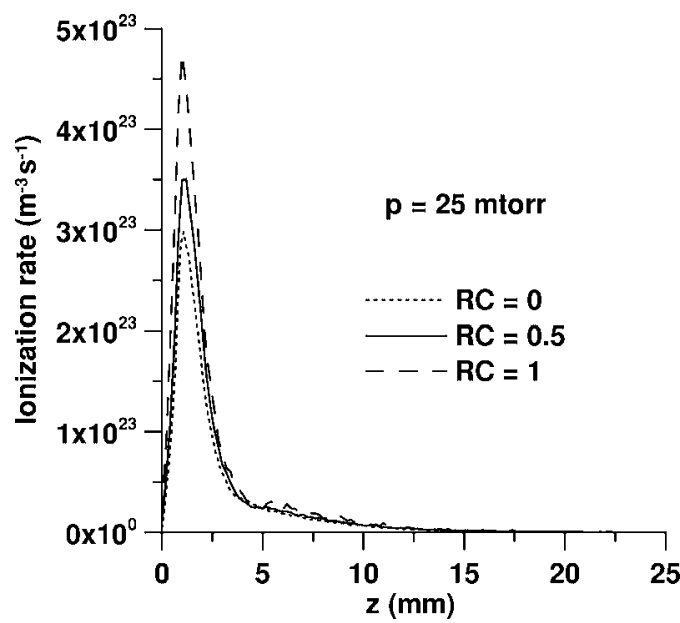

FIG. 11. Ionization rate along $r=17.2 \mathrm{~mm}$ at $p=25$ mtorr as a function of the reflection coefficient.

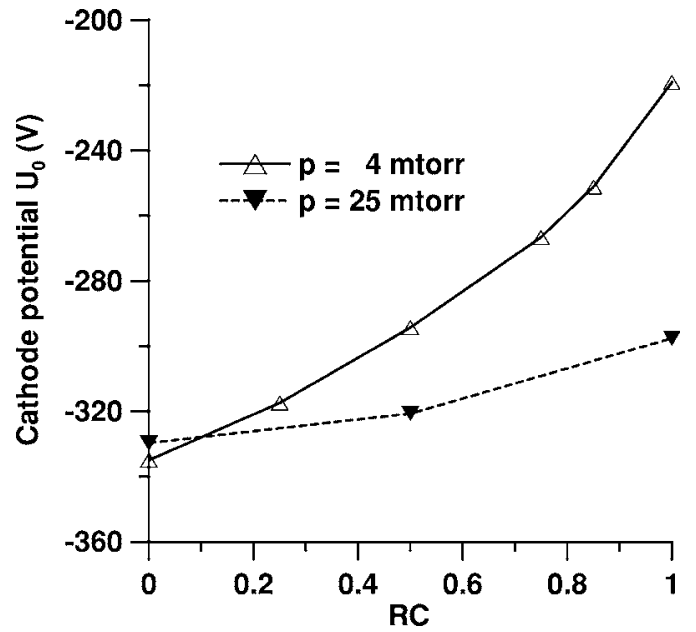

FIG. 12. Cathode potential $U_{0}$ as a function of the reflection coefficient. Solid curve for $p=4$ mtorr, dashed curve for $p$ $=25$ mtorr. Triangles represent the calculated values.

imagining a simple circuit consisting of a voltage source, a ballast resistor, and a variable resistor (representing the discharge) in series. When the resistance of the variable resistor is decreased, which exactly happens when the RC is increased, the current in the circuit increases, so does the voltage drop across the ballast resistor, and to obey Kirchhoff's voltage loop law, the discharge voltage must decrease. As can be seen at higher pressure, the change of $U_{0}$ and $I_{\text {disch }}$ with the RC is significantly weaker. The dependence is nonlinear and becomes stronger when allowing more reflected electrons. This is in contradiction with the statement made in [9], where the authors, performing a 1D PIC-MCC simulation of a cylindrical de magnetron, claim that no significant influence on the steady-state results is observed for RC between 0.5 and 0.9 .

\section{F. Effective SEEC}

It is interesting to give a macroscopic parameter, which can be used in analysis, with respect to the electron recap-

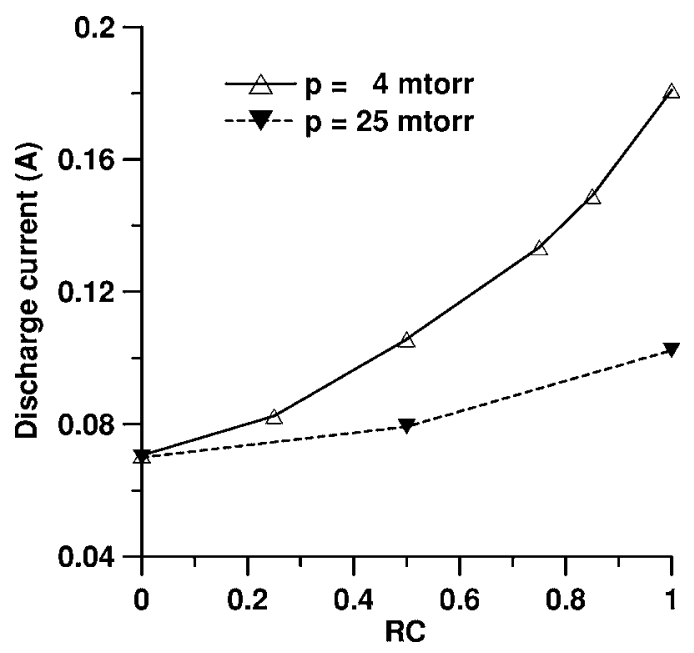

FIG. 13. Discharge current $I_{\text {disch }}$ as a function of the reflection coefficient. Solid curve for $p=4$ mtorr, dashed curve for $p$ $=25$ mtorr. Triangles represent calculated values. 


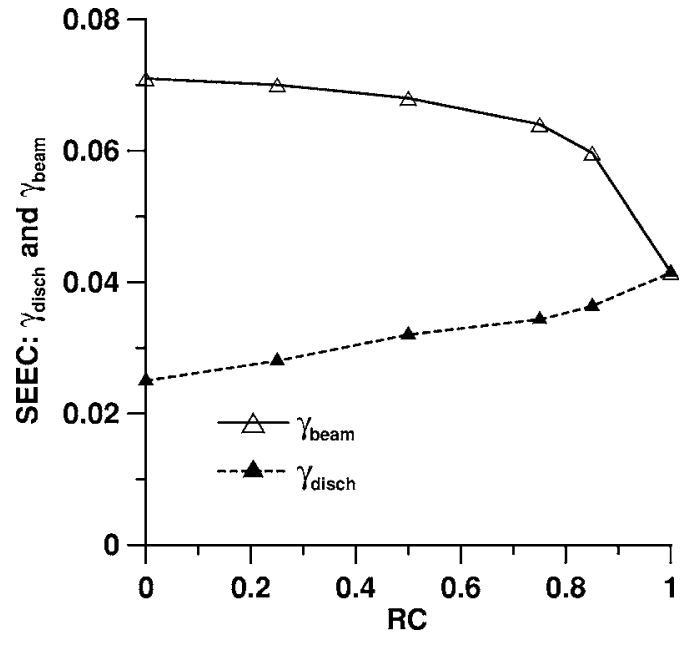

FIG. 14. Secondary-electron emission coefficient at $p=4$ mtorr as a function of the reflection coefficient. Solid curve: as seen by the cathode (SEEC from ion beam data). Dashed curve: as seen by the discharge (effective SEEC). Triangles represent calculated values.

ture. Such is the SEEC, but in the way the discharge sees it, $\gamma_{\text {disch }}$, which can be defined as

$$
\gamma_{\text {disch }}=\left(N_{e j}-N_{r e c}\right) / N_{i o n} .
$$

Here, $N_{e j}$ is the number of electrons produced by ion bombardment per unit time, $N_{\text {rec }}$ is the number of electrons recaptured at the cathode during this time, and $N_{i o n}$ is the number of ions striking the cathode for the same period of time. $\gamma_{\text {disch }}$ is, in fact, the effective SEEC, which should be used in estimates to account for the effect of electron recapture. The beam SEEC $\gamma_{\text {beam }}$, which is what is normally available from the literature, will be

$$
\gamma_{\text {beam }}=N_{e j} / N_{\text {ion }} .
$$

Both quantities are shown in Fig. 14, calculated at $p$ $=4$ mtorr for different RC values. As can be seen, the effective SEEC is significantly reduced due to the existence of electron recapture. For the intermediate value of $\mathrm{RC}=0.5$, $\gamma_{\text {disch }}$ is found to be exactly the half of $\gamma_{\text {beam }}$. This result confirms the assumption made by Thornton in [10] and is in good agreement with the value $\gamma_{\text {disch }}=0.46 \gamma_{\text {beam }}$ for a pressure of 3.75 mtorr obtained by Buyle et al. [11]. However, the exact value of $\gamma_{\text {disch }}$ is always a function of RC and pressure.

\section{G. Constant versus probabilistic recapture}

As has been mentioned in the Introduction and in Sec. III $\mathrm{C}$, the recapture of the electrons at the cathode can be treated according to Refs. $[17,18]$. The model described there is fully compatible with the PIC-MCC technique and capable of dealing with different kinds of surfaces. The surface properties are implemented in a set of parameters, which are derived from experimental data. Therefore, primal knowledge of experimental data for electron-induced secondary-electron emission is needed in order to apply the model. Beside this

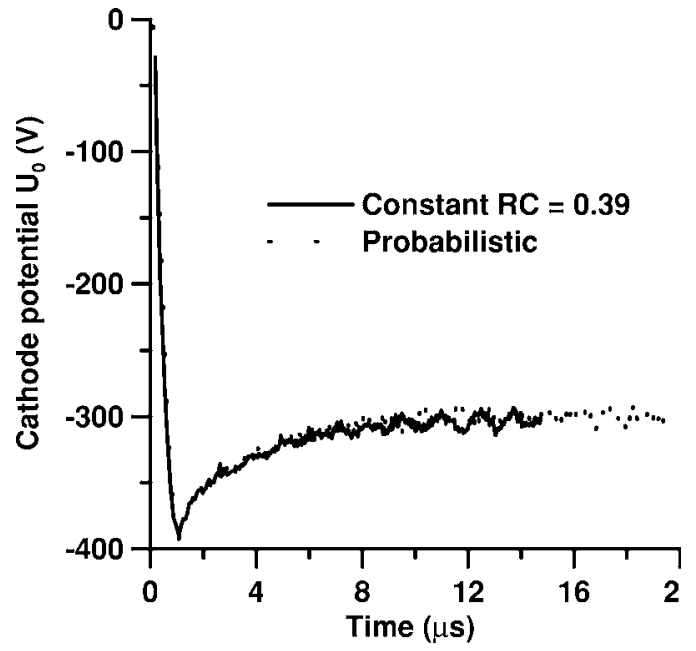

FIG. 15. Time evolution of the cathode potential. Dotted curve: recapture treated probabilistically, as in Refs. $[17,18]$. Solid curve: recapture treated with a constant $\mathrm{RC}=0.39$.

restriction, there exists a purely computational drawback. To obtain the probability for absorption, reflection, or emission of each electron hitting the cathode and the velocity of the reflected and emitted electrons, computationally exhaustive calculations have to be performed. This includes, among others, a numerical evaluation of normalized incomplete $\gamma$ and $\beta$ functions [43], as well as the functional inverse of both of them. In the present work, $\gamma$ and $\beta$ functions are calculated according to [44] and their functional inverse by applying the Newton-Raphson method [45].

A simulation with the above-mentioned algorithm has been run with the necessary parameters for $\mathrm{Cu}$. Next, a simulation with a constant $\mathrm{RC}=0.39$ has been carried out. The value 0.39 has been obtained as an averaged value from the first simulation. The results of both simulations are practically identical. An illustration of this statement is Fig. 15, where the temporal evolution of the cathode potential, $U_{0}$, for both cases is shown. As can be seen, in both experiments $U_{0}$ converges to a value of $-303 \mathrm{~V}$. The other discharge parameters (not shown here) exhibit also few differences. This is an expected result, having in mind that the electrons hitting the cathode have very narrow energy dispersion, which makes them beam like and with a low mean energy (a few eV), which makes the probability for an emission of a truly secondary electron statistically insignificant.

Therefore, using the probabilistic method for numerical simulation of dc magnetrons, although physically consistent and fully compatible with the PIC-MCC technique, could be successfully replaced by a constant RC value, provided that such a value is known from an experiment or theoretical calculations. The advantage of such a replacement is computational efficiency.

\section{CONCLUSION}

We have developed a two-dimensional particle-in-cellMonte Carlo code to investigate the possible effect of electron recapture at the cathode in planar dc magnetrons by 
means of numerical simulations. The results of the simulations indicate that electron recapture indeed happens and has a significant role in the processes in dc planar magnetrons. This role is manifested to be stronger at lower pressure (4 mtorr) than at higher pressure (25 mtorr). But since often magnetrons are operated at pressures even lower than 4 mtorr, the question of how the recapture is dealt with in models, theories, and estimates is essential for the correct understanding and prediction of these systems. The effect is not only pressure dependent, but also nonlinear with respect to the $\mathrm{RC}$ value. The $\mathrm{RC}$ can be used as a fitting parameter in numerical simulations in order to bring the calculated voltampere characteristics and electron densities in closer agreement to the experimentally measured values. We have also compared a simple approach based on the assumption of a constant RC with an approach based on a mathematically self-consistent phenomenological probabilistic model. The comparison shows that both approaches yield identical results and, therefore, the important issue for a correct numerical simulation of a dc magnetron is knowledge of the value of the RC.

\section{ACKNOWLEDGMENTS}

The authors want to thank to G. Buyle for providing the measured magnetic field data and erosion profile used in the present article and greatly appreciate useful discussions with A. Okhrimovskyy and U. Bhandarkar. This work has been financially supported by the Flemish Fund for Scientific Research (FWO, Flanders) and the IUAP V project.
[1] H. D. Hagstrum, Phys. Rev. 104, 672 (1956).

[2] F. F. Chen, Introduction to Plasma Physics and Controlled Fusion (Plenum Press, New York, 1984), Vol. 1, Chap. 2, p. 30.

[3] A. V. Phelps and Z. Lj Petrovich, Plasma Sources Sci. Technol. 8, R21 (1999).

[4] E. Shidoji, H. Ohtake, N. Nakano, and T. Makabe, Jpn. J. Appl. Phys., Part 1 28, 2131 (1999).

[5] S. Kondo and K. Nanbu, J. Phys. D 32, 1142 (1999).

[6] K. Nanbu, S. Segawa, and S. Kondo, Vacuum 47, 1013 (1996).

[7] J. Li and Z. Li, J. Phys. D 28, 1121 (1995).

[8] V. V. Serikov and S. Kawamoto (unpublished).

[9] T. A. van der Straaten, N. F. Cramer, I. S. Falconer, and B. W. James, J. Phys. D 31, 177 (1998).

[10] J. A. Thornton, J. Vac. Sci. Technol. 15, 171 (1978).

[11] G. Buyle, W. De Bosscher, D. Depla, K. Eufinger, J. Haemers, and R. De Gryse, Vacuum 70, 29 (2003).

[12] S. Anderson and B. Kasemo, Solid State Commun. 8, 961 (1970)

[13] E. G. McRae and C. W. Caldwell, Surf. Sci. 57, 77 (1976).

[14] A. J. Hatch and H. B. Williams, Phys. Rev. 112, 681 (1958).

[15] R. A. Kishek, Y. Y. Lau, L. K. Ang, A. Valfells, and R. M. Gilgenbach, Phys. Plasmas 5, 2120 (1998).

[16] R. Reimer, Scanning Electron Microscopy: Physics of Image Formation and Microanalysis, 2nd ed. (Springer-Verlag, Berlin, 1998).

[17] M. A. Furman and M. T. F. Pivi, PRST-AB 5, 124404 (2002).

[18] M. A. Furman and M. T. F. Pivi (unpublished).

[19] C. K. Birdsall and A. B. Langdon, Plasma Phyics via Computer Simulations (IOP, Bristol, 1991).

[20] C. K. Birdsall, IEEE Trans. Plasma Sci. 19, 66 (1991).

[21] J. Boris (unpublished).

[22] C. K. Birdsall and A. B. Langdon, Plasma Physics via Computer Simulations (IOP, Bristol, 1991), Appendix C, p. 437.

[23] H. R. Skullered, Br. J. Appl. Phys., J. Phys. D 1, 1967 (1968).

[24] K. Nanbu, IEEE Trans. Plasma Sci. 28, 971 (2000).

[25] K. Nanbu, Jpn. J. Appl. Phys., Part 1 33, 4752 (1994).

[26] R. W. Hockney and J. W. Eastwood, Computer Simulations Using Particles (IOP, Bristol, 1988), Vol. 1, Chap. 6, p. 199.
[27] P. N. Swartztrauber, SIAM (Soc. Ind. Appl. Math.) J. Numer. Anal. 11, 1136 (1974).

[28] V. Vahedi, C. K. Birdsall, M. A. Lieberman, G. DiPeso, and T. D. Rognlien, Phys. Fluids B 5, 2719 (1993).

[29] M. Hayashi (unpublished). A tabulation of these cross sections is available at ftp://jila.colorado.edu/collision_data/ electronneutral/hayashi.txt

[30] A. Okhrimovskyy, A. Bogaerts, and R. Gijbels, Phys. Rev. E 65, 037402 (2002).

[31] C. Opal, W. Peterson, and E. Beaty, J. Chem. Phys. 55, 4100 (1971).

[32] W. G. Vincenti and C. H. Kruger, Jr., Introduction to Physical Gas Dynamics (Kruger, Huntington, NY, 1977), Chap. IX, p. 352.

[33] A. V. Pheplps, J. Appl. Phys. 76, 747 (1994).

[34] A. V. Phelps (private communication). A review of the existing measurements and calculations of the cross sections for symetrical charge transfer is available at ftp://jila.colorado.edu/ collision_data/ionneutral/symmchgtransnote.txt

[35] M. Izawa, Y. Sato, and T. Toyomasu, Phys. Rev. Lett. 74, 5044 (1995)

[36] K. Ohmi, Phys. Rev. Lett. 75, 1526 (1995).

[37] V. Vahedi and G. DiPeso, J. Comput. Phys. 131, 149 (1996).

[38] J. C. Adam, A. G. Serveniere, and A. B. Langdon, J. Comput. Phys. 47, 229 (1982).

[39] J. W. Bradley, S. Thompson, and Y. Aranda Gonzalvo, Plasma Sources Sci. Technol. 10, 490 (2001).

[40] D. J. Field, S. K. Dew, and R. E. Burrell, J. Vac. Sci. Technol. A 20, 2032 (2002).

[41] N. Matsunami et al., At. Data Nucl. Data Tables 31, 1 (1984).

[42] G. Buyle (private communication).

[43] Handbook of Mathematical Functions, Natl. Bur. Stand. Appl. Math. Ser. No. 55, edited by M. Abramovitz and I. A. Stegun, (U.S. GPO, Washington, D.C., 1968), Vol. 55, Chap. 6.

[44] W. H. Press, S. A. Teukolsky, W. T. Vetterling, and B. P. Flannery, Numerical Recipes in Fortran 77: The Art of Scientifical Computing, 2nd ed. (Cambridge University Press, New York, 1996), Vol. 1, Chap. 6, pp. 209-221.

[45] F. S. Acton, Numerical Methods That Work (Mathematical Association of America, Washington, D.C., 1990), Chap. 2. 